\title{
Apoyo a la Autonomía, satisfacción de Necesidades Psicológicas Básicas, Motivación Autodeterminada y Bienestar en bailarines adolescentes de una escuela de danza.
}

\section{Autonomy Support, Satisfaction of Basic Psychological Needs, Self-Determined Motivation and Wellness in teenage dancers of a dance school.}

Fecha de recepción: 02/10/2016

Fecha de aceptación: 10/10/2016
Marta García Garay

Investigadora independiente y fundadora de Impulsarte Psicología

\section{resumen/alsstract:}

Tomando como base teórica la Teoría de la Autodeterminación (Deci y Ryan, 1985, 2000), se puso a prueba un modelo correlacional atendiendo a las variables: Apoyo a la Autonomía, satisfacción de Necesidades Psicológicas Básicas, Motivación Autodeterminada y Bienestar (Autoconcepto: Autovalía Global y Competencia en Danza). Muestra de 75 bailarines (Edad $M=12.49$; $D T=2.32$ ) la totalidad del alumnado adolescente de una escuela privada de danza. Se utilizaron diversos instrumentos estandarizados propios del deporte y adaptados a danza. Se realizaron estadísticos descriptivos, correlaciones de Pearson y análisis de la fiabilidad interna (Alfa de Cronbach), utilizando el paquete estadístico SPSS 21. Los índices de fiabilidad superaron los criterios recomendables $(\alpha>.70)$ a excepción de la Competencia en Danza $(\alpha=.46)$. Los resultados ofrecieron apoyo parcial al modelo propuesto. Cuando los bailarines perciben que sus profesores les proporcionan un clima de apoyo a la autonomía, informan de mayor satisfacción de sus necesidades psicológicas (especialmente autonomía y relación) y de mayor calidad de la experiencia: mayor motivación autodeterminada hacia el baile. No se han confirmado las hipótesis relacionadas con el autoconcepto.

Taking the Self-Determination Theory as a basis (Deci and Ryan, 1985, 2000) a correlational model was tested attending to the variables: Autonomy support, Satisfaction of basic psychological needs, Self-determined motivation and Wellness (Self-concept: Global self-worth and Dance Competence). Sample of 75 dancers (Age M=12.49; DT=2.32) the totality of the teenage student body from a private dance school. Diverse standardized instruments likely to be used in sport and adapted to dance were employed. Descriptive statistics, Pearson correlations, and internal reliability analysis (Cronbach's Alfa) were made, using the statistic package SPSS 21. The reliability indexes overcame the recommended criteria $(\alpha>$.70) excluding Dance Competence ( $\alpha=46$.) The results offered partial support to the proposed model. When the dancers notice that their teachers provide an atmosphere of autonomy support, they result in a higher satisfaction of their psychological needs (specially autonomy and relation) and a higher quality of the experience: bigger self determined motivation towards dance. Hypothesis related to self-concept have not been confirmed.

\section{palabras clave/keywords:}

Teoría de la Autodeterminación, Danza, Psicología de la Danza, Psicología Deportiva, Clima, Bienestar. Self-Determination Theory, Dance, Dance Pshychology, Sports Physchology, Climate, Wellness. 
La motivación, en su sentido más amplio, implica la habilidad de emprender una tarea y persistir en ella. Aplicada a la danza, podría definirse como "la capacidad de los bailarines para persistir ante el aburrimiento, la fatiga, el dolor y el deseo de hacer otras cosas" (Taylor y Taylor, 2008; p.41). Tanto los bailarines como sus mentores (padres, profesores y entrenadores) buscan la forma de movilizar esfuerzos hacia una misma meta (Taylor y Taylor, 2008).

La danza, pese a ser una disciplina artística, comparte características con contextos deportivos (entrenamientos/ensayos, figura del entrenador/profesor, pertenencia al club/academia, partidos/ actuaciones, relación con los compañeros, etc.). Por este motivo, la Psicología del Deporte se interesa cada vez más por conocer qué particularidades del entorno de la danza son las adecuadas para que los bailarines se desarrollen de forma óptima (Taylor y Taylor, 2008).

Una de las teorías motivacionales que tiene como objetivo el desarrollo positivo de las personas es la Teoría de la Autodeterminación (Deci y Ryan, 1985, 2000) (SDT por sus siglas en inglés), la cual sostiene que los individuos son organismos activos con tendencias innatas hacia superar los retos ambientales y a implicarse eficazmente en el entorno. El contexto social puede apoyar o frustrar las tendencias naturales hacia la participación activa y el crecimiento psicológico. Si en su interacción con el medio las personas regulan sus conductas de forma voluntaria y volitiva, se favorecerá la calidad de la implicación y el bienestar (Ryan y Deci, 2000b, 2002). Esta teoría nos ayuda a detectar las razones por las que los bailarines están implicados en la danza en forma de regulaciones motivacionales más o menos autodeterminadas (Balaguer, Castillo, Duda, y García-Merita, 2011). Se basa en los siguientes conceptos: Clima Motivacional de Apoyo a la Autonomía, Regulaciones Motivacionales, Teoría de las Necesidades Psicológicas Básicas (BPNT, Basic Psychological Needs Theory) que son Autonomía, Competencia y Relación, y Bienestar. En el apartado de método se explicarán brevemente estos conceptos. Para ahondar más en ellos se recomienda al lector que consulte los trabajos de: Deci y Ryan (1985, 2000); Ryan y Deci (2000a, 2000b, 2002, 2007).

Se ha tomado el Autoconcepto como medida de bienestar psicológico. En concreto, este trabajo se basa en el marco teórico proporcionado por Susan Harter (1999). Harter (1999) utiliza un enfoque de "automejora" (autoevaluación distorsionadamente positiva), considerando que los individuos están motivados a actuar en las áreas en las que es probable que experimenten sentimientos positivos de competencia y estima. Por otro lado, mostrarán amotivación hacia actividades propias de áreas en las que no se sientan competentes (Balaguer y Pastor, 2001).

La danza es un ámbito poco estudiado desde la psicología si lo comparamos con otros deportes (Taylor y Taylor, 2008). La trayectoria de Eleonor Quested y Joan L. Duda aporta una línea de investigación con interesantes resultados. Han estudiado la influencia de los factores que conforman la SDT sobre otras variables: cambios en estados afectivos (Quested, Duda, Ntoumanis, y Maxwell, 2013), respuestas hormonales y emocionales (Quested et al, 2010), tendencias de autoevaluación (Quested y Duda, 2009a) y percepción física 
(Quested y Duda, 2011). Se ha comprobado que la percepción de apoyo a la autonomía y la satisfacción de las necesidades psicológicas básicas ayuda a evitar el burnout (síndrome de "estar quemado") en bailarines profesionales (Quested y Duda, 2011), es un predictor de la intención de seguir participando (Balaguer et al, 2011) y promueve experiencias de entrenamiento óptimo (Quested y Duda, 2009b). En el reciente trabajo de González, Castillo, García-Merita y Balaguer (2015) se comprueba la secuencia teorizada por la SDT entre apoyo a la autonomía, satisfacción de las necesidades psicológicas y bienestar (en concreto, medidas de vitalidad subjetiva y afecto positivo), revelando la invarianza del modelo entre dos disciplinas deportivas: danza y futbol. Guzmán y García-García (2012), investigaron acerca del bienestar psicológico en bailarines desde una perspectiva socio-cognitiva, partiendo de la SDT.

En base a los postulados teóricos e investigaciones previas, el principal objetivo de esta investigación es estudiar la secuencia que aparece en la Figura 1 en la totalidad de bailarines adolescentes que acuden a un mismo centro privado de danza.

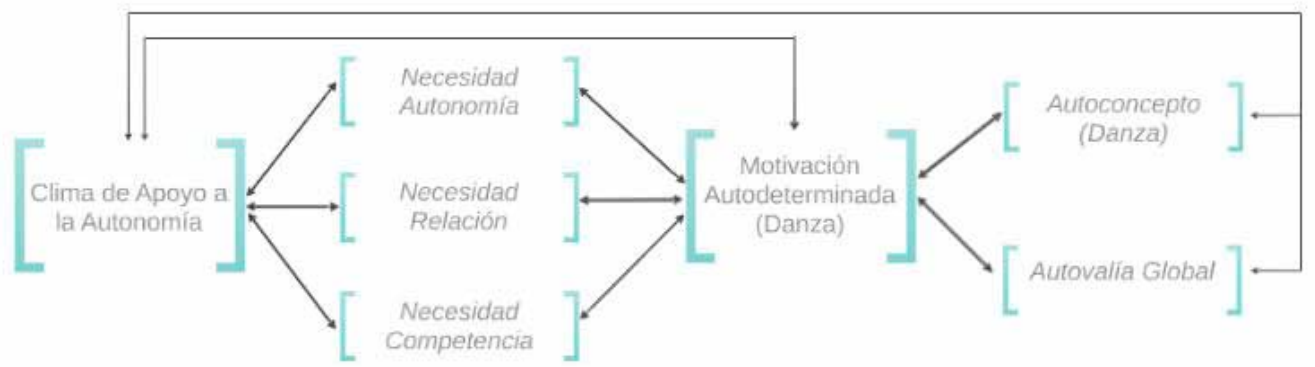

Correlación positiva:

Figura 1: Modelo a comprobar. Correlaciones entre las dimensiones estudiadas.

Se espera que (1) una elevada percepción de apoyo a la autonomía en el centro de danza esté relacionada con una alta satisfacción de las necesidades psicológicas básicas: Autonomía, Relación y Competencia. (2) La satisfacción de las necesidades psicológicas básicas se relacione con altos niveles de motivación autodeterminada en danza y que (3) una elevada percepción de apoyo a la autonomía en el centro de danza correlacione significativamente con una elevada motivación autodeterminada en danza. Por otro lado, (4) se espera que una elevada motivación autodeterminada en danza se vincule con índices de bienestar mayores. Por último, (5) que elevada percepción de apoyo a la autonomía en el centro de danza correlacione significativamente con altos niveles de bienestar.

\section{Método}

\section{Participantes}

Se procedió a la recogida de datos correspondientes a la totalidad de alumnos de una escuela de danza de la localidad de Massanassa (Valencia) por petición de la dirección de dicho 
centro, que esperaba utilizar este estudio para enfocar la planificación de la formación de su profesorado. En total participaron 75 adolescentes (10 chicos y 65 chicas) con edades comprendidas entre los 8 y los 16 años $\left(_{E d a d} M=12,49\right.$ años; $\left.D T=2.32\right)$ que reciben clase en el mismo centro. El $46.7 \%$ de estos bailarines aprenden varios estilos. El $89.3 \%$ tiene más de una clase a la semana, por lo tanto, ha basado sus respuestas en una visión global de la academia, teniendo en cuenta su relación con varios profesores.

El 38\% de la muestra participa en competiciones de baile deportivo o danza urbana. Los estilos más bailados son el Hip Hop y el Funky, englobados dentro de la categoría "Danza Urbana" (85.3\%). Por otro lado, el menos demandado es la Salsa (8\%), más enfocada a la población adulta. Otros estilos ofrecidos por esta escuela son Danza Clásica (16\%) y Danza Contemporánea (24\%).

\section{Instrumentos}

Se utilizaron auto-informes validados en el ámbito del deporte, adaptando la redacción de la frase introductoria y de los diferentes ítems al ámbito de la danza. Se describirán a continuación de forma detallada:

El Clima Motivacional de Apoyo a la Autonomía hace referencia a los agentes de socialización (en este caso, profesores de baile), que promueven la internalización de las acciones y una motivación autodeterminada regulada (Deci y Ryan, 2000). Se evaluó mediante la versión castellana (Balaguer, Castillo, Duda, y Tomás, 2009) del Cuestionario de Clima en el Deporte (SCQ, Sport Climate Questionnaire, n.d.). Se ha utilizado la versión larga de esta escala, que consta de 15 ítems. Evalúa el grado en que los bailarines perciben que su profesor de danza apoya su autonomía en el baile. Cada ítem se inicia con la frase: "En esta escuela de danza" y las respuestas se recogen en una escala tipo Likert que oscila desde totalmente en desacuerdo (1) hasta totalmente de acuerdo (7). Un ejemplo de ítem sería: "Mis profesores de danza hacen que yo confíe en mi habilidad para bailar bien". La fiabilidad de la escala original obtuvo un $\alpha=.95$ (Williams, Grow, Freedman, Ryan y Deci, 1996) y la versión adaptada por Balaguer et al (2009) obtuvo un $\alpha=.96$. Otras investigaciones han confirmado la fiabilidad de esta escala con deportistas (Balaguer, Castillo, y Duda, 2008; Balaguer et al, 2009) y con practicantes de danza (Balaguer, Castillo, Duda, Quested, y Morales, 2011; Quested y Duda, 2010, 2011).

La conocida como BPNT (Basic Psychological Needs Theory), es una de las mini-teorías que componen la SDT. Estipula que el efecto del medio ambiente sobre las bases de la conducta no es directo, sino que se produce como resultado de la satisfacción de las tres necesidades psicológicas básicas: autonomía, competencia y relación. Éstas se consideran "nutrientes psicológicos innatos esenciales para el crecimiento psicológico en curso, la integridad y el bienestar." (Deci y Ryan, 2000). La satisfacción de autonomía se define como la participación volitiva en acciones, siendo coherente con valores e intereses personales (DeCharms, 1968). Se considera que se satisface la necesidad de competencia cuando la persona detecta que está operando de manera eficaz en el medio y tiene la capacidad de lograr los resultados deseados (White, 1959). Por último, la satisfacción de relación se refiere a sentirse conectado con los otros, logrando relaciones interpersonales significativas y ape- 
go social, generando así un sentimiento de pertenencia. La satisfacción de las necesidades psicológicas básicas se midió con los instrumentos que se detallan a continuación:

La Escala de Competencia Percibida del Cuestionario de Motivación Intrínseca (McAuley, Duncan, y Tammen, 1989) está compuesta por cinco ítems que evalúan la competencia percibida en el contexto deportivo. Se utilizó la versión adaptada por Balaguer et al (2008). Se solicitó a los bailarines que indicasen el nivel de acuerdo con una serie de afirmaciones como "Soy bastante bueno (habilidoso) en la danza". Las respuestas se recogen en una escala tipo Likert de siete puntos, que oscila desde totalmente en desacuerdo (1), hasta totalmente de acuerdo (7). La fiabilidad de la escala original de McAuley et al (1989) obtuvo una fiabilidad satisfactoria con $\alpha=.80$, por su parte la versión adaptada por Balaguer et al (2008) obtuvo un $\alpha=.79$

La Escala de Autonomía Percibida en el Deporte elaborada por Reinboth y Duda (2006) nos permite evaluar dos facetas de la autonomía percibida: la elección/toma de decisión, con 4 ítems y los aspectos volitivos en el contexto deportivo, con 6 ítems. Su fiabilidad obtuvo un $\alpha=.87$ y $\alpha=.80$, respectivamente. Se utilizó la versión adaptada por Balaguer et al (2008) cuya fiabilidad fue satisfactoria $\alpha=.89$. En sus 10 ítems se pide a los participantes que indiquen cómo se sienten en general cuando participan en su disciplina, por ejemplo: "En mi escuela de danza... me siento libre de expresar mis ideas y mis opiniones". Las respuestas se recogen en una escala tipo Likert de siete puntos, con un rango desde nada verdadero (1), hasta muy verdadero (7).

La subescala de Aceptación de la Escala de Necesidad de Relación de Richer y Vallerand (1998) está compuesta por cinco ítems que evalúan el nivel de aceptación y respeto percibido en el contexto deportivo. La escala original de Richer et al (1998) obtuvo una fiabilidad satisfactoria con $\alpha=.89$. Se utilizó la versión adaptada por Balaguer et al (2008), que obtuvo un $\alpha=.87$. En esta escala se solicita a los participantes que indiquen el nivel personal de acuerdo a cómo se sienten cuando practican su actividad, por ejemplo: "En esta escuela de danza me siento apoyado/a". Los bailarines contestaron en una escala tipo Likert de cinco puntos, con un rango desde totalmente en desacuerdo (1), hasta totalmente de acuerdo (5).

Las Regulaciones Motivacionales se refieren al por qué de la conducta, es decir, al rango de motivos para la acción en todos los dominios de la vida. Las regulaciones de la conducta pueden situarse a lo largo de un continuo de autodeterminación: desde la no motivación o amotivación, pasando por estilos de regulación controlados (regulación externa y regulación introyectada) hasta formas autodeterminadas de regulación (regulación identificada, regulación integrada y motivación intrínseca).

La Motivación en la práctica de la danza fue evaluada con la versión castellana adaptada a la danza (Balaguer et al, 2011) del Cuestionario de Regulación Conductual en el Deporte (Lonsdale, Hodge, y Rose, 2008). Está compuesto por 24 ítems, divididos en seis subescalas en las que se solicita a los bailarines que piensen sobre los motivos por los que participan en danza. A continuación se presentan ejemplos de ítems para cada una de las subescalas, iniciados con la frase "Yo participo en danza...": "Porque disfruto haciéndolo" (motivación intrínseca), "Porque es una oportunidad para ser quien soy" (regulación inte- 
grada), "Porque los beneficios que recibo de la danza son importantes para mi" (regulación identificada),"Porque me sentiría fracasado/a si lo dejase" (regulación introyectada), "Con la finalidad de satisfacer a las personas que quieren que yo baile" (regulación externa),"Pero me pregunto por qué sigo" (amotivación). Las respuestas se recogen en una escala tipo Likert que oscila desde nada verdadero (1), hasta completamente verdadero (7). Los coeficientes de fiabilidad de las subescalas fueron satisfactorios tanto en la versión original de Lonsdale et al (2008) con alphas entre $\alpha=.79$ y $\alpha=.93$, como en la adaptación de Balaguer et al (2011) con alphas entre $\alpha=.74$ y $\alpha=.95$.

La última variable del estudio se refiere al Autoconcepto, como forma de medir el Bienestar Psicológico. Concretamente, se ha utilizado el Perfil de Autopercepción de Harter (Harter, 1985). El cuestionario completo evalúa seis dominios de la autopercepción: competencia académica, aceptación social, competencia deportiva, apariencia física, comportamiento y autovalía global. Para este trabajo se han utilizado dos de estas dimensiones. La Autovalía Global, que se define como el grado en que el individuo se agrada como persona y está feliz con la forma en que conduce su vida. La Competencia Deportiva es la capacidad para los deportes o, en este caso, la práctica de la danza. Cada subescala está formada por 6 ítems. Ejemplos de ellos serían: "Algunas personas creen que son buenas haciendo todo tipo de danza /sin embargo/ otras personas creen que no son buenas cuando se trata de danza" (competencia en danza); "Algunas personas se enfadan con ellas mismas con frecuencia / sin embargo/ otras están bastante contentas con ellas mismas" (autovalía global). Los sujetos tienen que decidir con cuál de los dos grupos que aparecen descritos se sienten más identificados. A continuación marcan en qué grado se da tal similitud ("realmente como yo" o "parcialmente como yo"). Los coeficientes de fiabilidad fueron satisfactorios: competencia deportiva con un $\alpha=.84$ y autovalía global con un $\alpha=.84$. (Harter, 2012).

\section{Procedimiento}

Para solicitar la participación de los alumnos en este estudio de diseño correlacional se redactó un documento informativo explicando el objetivo de la investigación, así como un consentimiento informado que fue firmado por los tutores legales de los sujetos, de acuerdo con las normas éticas pertinentes. La recogida de datos se realizó en la academia, al inicio de cada clase durante una semana del mes de diciembre. Los grupos eran de 15 personas, y la administración tuvo una duración aproximada de 20 minutos. Se pidió a los alumnos que completaran los cuestionarios pensando en todos sus profesores y disciplinas. Cada paquete de cuestionarios fue revisado por el administrador previamente a la recogida del mismo, de modo que se comprobó que todos los ítems estaban cumplimentados de forma correcta.

\section{Análisis de Datos}

Se utilizó el paquete estadístico SPSS 21 para examinar los resultados. Se realizaron estadísticos descriptivos, análisis de la fiabilidad interna mediante el coeficiente de Alfa de Cronbach y correlaciones de Pearson.

\section{Resultados}

En la Tabla 1 se presentan los estadísticos descriptivos de las escalas utilizadas en el estu- 
dio. Los bailarines perciben que en su escuela de danza se crea un alto clima de apoyo a la autonomía $(M=6.11 ; D T=.56)$. Los participantes informan tener satisfechas sus necesidades psicológicas básicas (especialmente la necesidad de Relación, $M=4.44 ; D T=.75$ ). En el continuo de Autodeterminación, se puede afirmar que los bailarines se sienten motivados de forma intrínseca $(M=6.71 ; D T=.71)$. En cambio, las puntuaciones en Autoconcepto, Autovalía Global y Autopercepción de Competencia en Danza, no son especialmente elevadas (véase Tabla 1).

Tabla 1. Estadísticos descriptivos y análisis de la fiabilidad SDT. N=75.

\begin{tabular}{lcccc}
\hline Variables & Rango & Media & D.T & Alfa \\
\hline Clima de Apoyo a la Autonomía & $1-7$ & 6.11 & .56 & .79 \\
\hline Satisfacción Necesidad Autonomía & $1-7$ & 5.24 & 1.37 & .86 \\
\hline Satisfacción Necesidad de Relación & $1-5$ & 4.44 & .75 & .89 \\
\hline Satisfacción Necesidad de Competencia & $1-7$ & 5.51 & 1.01 & .70 \\
\hline Motivación Intrínseca Danza & $1-7$ & 6.71 & .57 & .71 \\
\hline Regulación Integrada Danza & $1-7$ & 6.24 & .92 & .76 \\
\hline Regulación Identificada Danza & $1-7$ & 6.06 & 1.01 & .76 \\
\hline Regulación Introyectada Danza & $1-7$ & 2.91 & 1.58 & .67 \\
\hline Regulación Extrínseca Danza & $1-7$ & 2.03 & 1.28 & .71 \\
\hline Amotivación Danza & $1-7$ & 2.20 & 1.43 & .76 \\
\hline Autovalía Global & $1-4$ & 1.98 & .63 & .69 \\
\hline Competencia en Danza & $1-4$ & 2.52 & .52 & .46 \\
\hline
\end{tabular}

Se comentan los coeficientes de fiabilidad de las pruebas utilizadas que aparecen en la Tabla 1. La mayor parte de los factores ha obtenido coeficientes de Alfa de Cronbach satisfactorios, ya que se encuentran por encima del criterio .70 determinado para las escalas del dominio psicológico (Nunnally, 1978). Estos índices pueden crecer cuando se aumenta el número de ítems que componen la escala, por lo que se argumenta que un coeficiente de .60 se consideraría aceptable en el caso de escalas compuestas por un número reducido de ítems, como es el caso de los factores utilizados (Hair, Black, Babin, Anderson, y Tatham, 2006). 
Las dimensiones que componen el cuestionario Harter (1985) referidas a la autopercepción de competencia en danza no han alcanzado los índices de fiabilidad esperables en nuestra muestra, por lo que los análisis correlacionales deberán interpretarse con cautela.

Observando las características de la muestra mediante el estadístico correlación de Pearson, se ha hallado una correlación significativa entre el número de estilos que practican los alumnos y: la cantidad de profesores $(0.901 * *)$ y su antigüedad en la academia $\left(0.389^{* *}\right)$. La correlación de Pearson muestra significación entre la antigüedad en la academia y el hecho de competir $(0.593 * *)$.

Pasamos a comentar las correlaciones bivariadas del modelo a comprobar. La Tabla 2 muestra las correlaciones entre Apoyo a la Autonomía, Satisfacción de las Necesidades Psicológicas Básicas y Regulaciones Motivacionales en Danza. La percepción de apoyo a la autonomía por parte de los bailarines hacia la escuela de danza presenta una correlación positiva con la satisfacción de autonomía y de relación. Las satisfacciones de las tres necesidades psicológicas básicas se asocian positivamente entre sí con una correlación significativa. Existen correlaciones significativas entre el apoyo a la autonomía percibido y las formas más autodeterminadas de regulación en Danza. Se observan altas correlaciones entre los factores que componen esta variable. La satisfacción de la necesidad de autonomía correlaciona con una de las formas de regulación motivacional más autodeterminadas: la regulación identificada. La satisfacción de la necesidad de relación se asocia positiva y significativamente con la motivación intrínseca y con la regulación identificada en el ámbito de la danza.

Tabla 2. - Correlaciones bivariadas entre Apoyo a la Autonomía y Satisfacción de las Necesidades Psicológicas Básicas y Regulaciones motivacionales en Danza. N=75.

\begin{tabular}{lllllllllll}
\hline Variables: & 1 & 2 & 3 & 4 & 5 & 6 & 7 & 8 & 9 & 10 \\
\hline 1. Apoyo Autonomía & 1 & .149 &. $\mathbf{7 1 3 * *}$ & $\mathbf{. 5 3 9 * *}$ &, $\mathbf{4 2 0 * *}$ &, $244^{*}$ & $\mathbf{2 6 4 *}$ &, 014 &,- 025 &,- 142 \\
2. Nec. Competencia & .149 & 1 & $.311^{* *}$ & $.340^{* *}$ &, 018 &, 216 &, 172 &, 265 &,- 011 &, 113 \\
3. Nec. Relación & $.713^{* *}$ & $.311^{* *}$ & 1 & $.622^{* *}$ &, $411^{* *}$ &, 110 &, $233^{*}$ &,- 040 &,- 85 &,- 110 \\
4. Nec. Autonomía & $.539^{* *}$ & $.340^{* *}$ & $.622^{* *}$ & 1 &, 209 &, 158 &, $327^{* *}$ &,- 014 &,- 033 &,- 060 \\
5. Mot. Intrínseca &, $420^{* *}$ &, 018 &, $411^{* *}$ &, 209 & 1 &, $306^{* *}$ &, $456^{* *}$ &, 024 &,- 059 &,- 191 \\
6. Reg. Integrada &, $244^{*}$ &, 216 &, 110 &, 158 &, $306^{* *}$ & 1 &, $417^{* *}$ &, $314^{* *}$ &, 181 &,- 019 \\
7. Reg. Identificada &, $264^{*}$ &, 172 &, $233^{*}$ &, $327^{* *}$ &, $456^{* *}$ &, $417^{* *}$ & 1 &, $305^{* *}$ &, 048 &, 048 \\
8. Reg. Introyectada &, 014 &, 265 &,- 040 &,- 014 &, 024 &, $314^{* *}$ &, $305^{* *}$ & 1 &, $436^{* *}$ &, $460^{* *}$ \\
9. Reg. Extrínseca & $-0,25$ &,- 011 &,- 85 &,- 033 &,- 059 &, 181 &, 048 &, $436^{* *}$ & 1 &, $647^{* *}$ \\
10. Amotivación &,- 142 &, 113 &,- 110 &,- 060 &,- 191 &,- 019 &, 048 &, $460^{* *}$ &, $674^{* *}$ & 1 \\
\hline
\end{tabular}

** $\mathrm{P}<0.01 ; * \mathrm{P}<0.05$ 
Como se ha comentado, el cuestionario de autoconcepto propuesto por Harter está conformado por seis facetas. En este trabajo se han utilizado dos de ellas, por ello se han querido calcular las correlaciones entre dichas dimensiones, obteniendo relación significativa $(.331 * *)$.

En la Tabla 3 se presentan los resultados del análisis de correlaciones entre las regulaciones motivacionales en danza y las medidas de autovalía global y autopercepción de competencia en danza. Han aparecido relaciones significativas negativas entre las medidas más autorreguladas de motivación en danza y la autovalía global, destacando las correlaciones entre motivación integrada e identificada en danza y autovalía global.

Tabla 3. - Correlaciones bivariadas entre las dimensiones de Autovalía Global, Competencia en danza y Regulaciones motivacionales en danza. $\mathrm{N}=75$.

\begin{tabular}{lllllllll}
\hline Variable & 1 & 2 & 3 & 4 & 5 & 6 & 7 & 8 \\
\hline 1. Autovalía Global & 1 & $.331^{* *}$ & $-.246^{*}$ & $\mathbf{- . 3 5 1 * *}$ & $-.317^{* *}$ & .029 & .028 & .180 \\
2. Competencia Danza & $.331^{* *}$ & 1 & .091 & -.171 & .016 & -.202 & .031 & -.104 \\
3. Mot. Intrínseca & $-.246^{*}$ & .091 & 1 & $.306^{* *}$ & $.456^{* *}$ & .024 & -.059 & -.191 \\
4. Reg. Integrada & $-.351^{* *}$ & -.171 & $.306^{* *}$ & 1 & $.417^{* *}$ & $.314^{* *}$ & .181 & -.019 \\
5. Reg. Identificada & $-.317^{* *}$ & .016 & $.456^{* *}$ & $.417^{* *}$ & 1 & $.305^{* *}$ & .048 & .048 \\
6. Reg. Introyectada & .029 & -.202 & .024 & $.314^{* *}$ & $.305^{* *}$ & 1 & $.436^{* *}$ & $.460^{* *}$ \\
7. Reg. Extrínseca & .028 & .031 & -.059 & .181 & .048 & $.436^{* *}$ & 1 & $.674^{* *}$ \\
8. Amotivación & .180 & -.104 & -.191 & -.019 & .048 & $.460^{* *}$ & $.674^{* *}$ & 1 \\
\hline
\end{tabular}

** $\mathrm{P}<0.01 ; * \mathrm{P}<0.05$

En cuanto a la relación entre la percepción de apoyo a la autonomía de los bailarines y las dimensiones de autovalía y competencia en danza, la única correlación significativa ha sido negativa y se ha hallado entre apoyo a la autonomía y autovalía global (Tabla 4).

Se presenta a continuación el modelo a comprobar, incorporando las correlaciones significativas halladas.

Tabla 4. - Correlaciones bivariadas entre las dimensiones de Autovalía Global, Competencia en Danza y Apoyo a la Autonomía. N=75.

\begin{tabular}{llll}
\hline Variable & 1 & 2 & 3 \\
\hline 1. Apoyo a la autonomía & 1 & $-.298 * *$ & -.89 \\
2. Autovalía Global & $-.298^{* *}$ & 1 & $.331 * *$ \\
3. Competencia en Danza & -.89 & $.331^{* *}$ & 1 \\
\hline$* * \mathrm{P}<0.01 ; * \mathrm{P}<0.05$ & & &
\end{tabular}




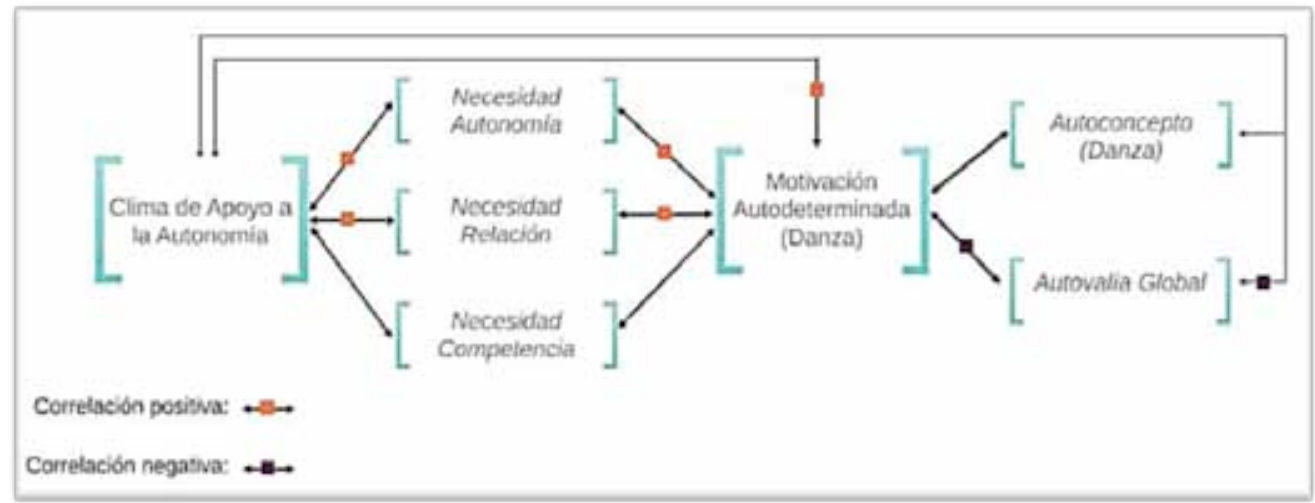

Figura 2: Modelo corregido de correlaciones significativas positivas y negativas entre las dimensiones estudiadas.

\section{Discusión}

En base a la SDT, el presente trabajo estudia las correlaciones entre apoyo a la autonomía, satisfacción de necesidades psicológicas básicas, motivación autodeterminada y bienestar en la totalidad de alumnado adolescente de una escuela de danza. Los resultados ofrecieron apoyo parcial al modelo propuesto.

La primera relación a comprobar es la establecida entre el clima de apoyo a la autonomía y la satisfacción de las necesidades psicológicas básicas del alumnado de danza (hipótesis 1). En consonancia con los postulados teóricos, aquellos participantes que perciben mayor apoyo a la autonomía ofrecido por la escuela de baile afirman disfrutar de mayores niveles de satisfacción de sus necesidades de autonomía y relación. Cuando se perciben alternativas de elección por parte de las figuras de autoridad, los alumnos se sienten comprendidos y, por tanto, es más probable que consideren que ellos mismos son la causa de sus propias acciones (de Charms, 1968). Cuando los deportistas perciben que su entrenador se comporta de forma empática y se sienten comprendidos y apoyados, se sienten también mutuamente valorados por sus compañeros y se ven favorecidas sus relaciones sociales (Balaguer et al, 2008). La correspondencia entre el clima de apoyo a la autonomía y la satisfacción de la necesidad de competencia resultó positiva aunque no significativa en el presente estudio, lo cual podría explicarse por las características de la muestra. Se trata de adolescentes pertenecientes a una escuela de danza a la que muchos alumnos acuden como forma de ocio y no en todos los grupos se fomenta el rendimiento tanto como en conservatorios o niveles de élite. Otras investigaciones avalan estos resultados como por ejemplo, Balaguer et al (2008) en una muestra de deportistas. El trabajo de González, Tomás, Castillo, Duda y Balaguer (2016) muestra que la percepción de apoyo a la autonomía proporcionada por el entrenador predijo la satisfacción de la necesidad de autonomía y relación en el equipo, mientras que los resultados a nivel individual mostraron que la percepción de apoyo a la autonomía del entrenador predijo positivamente la satisfacción de las tres necesidades psicológicas 
básicas. En el presente estudio se ha hallado relación significativa entre la condición de participar en competiciones y las puntuaciones obtenidas en la satisfacción de la necesidad de competencia de esta muestra. En el trabajo de Quested y Duda (2011) realizado con una muestra de bailarines de élite sí se confirmaron las relaciones entre el apoyo a la autonomía y la satisfacción de las tres necesidades. El estudio de González, Castillo, García-Merita y Balaguer (2015) con una muestra de bailarines y futbolistas demuestra que la percepción de apoyo a la autonomía ofrecido por el profesor/entrenador predice de forma positiva la satisfacción de las necesidades psicológicas básicas. Estos resultados son congruentes con estudios previos en diferentes deportes (Adie Duda y Ntoumanis, 2008, 2012; LópezWalle, Balaguer, Castillo y Tristán, 2012).

La siguiente parte del modelo supone una relación positiva significativa entre la satisfacción de las necesidades psicológicas básicas en el centro de danza y el continuo de regulaciones autodeterminadas en danza (hipótesis 2). En nuestro trabajo, los bailarines que tienen más satisfechas sus necesidades de autonomía y relación, gozan de una motivación más intrínseca hacia la actividad de bailar, confirmando lo esperado. Esto resulta muy positivo para su persistencia en la actividad (Balaguer et al, 2011) y previene el burnout (Quested y Duda, 2011). De nuevo, los resultados referentes a la satisfacción de competencia van en la línea prevista pero no resultan significativos. Posiblemente los motivos sean los anteriormente nombrados. Los bailarines que tienen más satisfechas sus necesidades psicológicas utilizan en mayor medida estrategias de afrontamiento orientadas a la tarea (Amado, Leo, Sánchez, Sánchez, y García-Calvo, 2010).

Centrándonos en los supuestos de la SDT, es de esperar que cuando la academia de baile proporciona un clima positivo (de apoyo a la autonomía) a sus alumnos, éstos experimenten un mayor grado de regulación autodeterminada en la actividad de bailar (hipótesis 3). Los profesores deben transmitir sentimientos y conductas dirigidos a detectar, favorecer y desarrollar recursos motivacionales internos, intereses personales, metas intrínsecas y valores propios de cada uno de los bailarines (Reeve, 2009). Los resultados del presente trabajo confirman dicha relación, incluso en el polo más positivo del continuo de autoregulación: la motivación intrínseca. En investigaciones realizadas en el contexto del deporte como las de Balaguer et al, (2009) o Pelletier, Fortier, Vallerand, y Brière (2001), los deportistas afirmaron que cuando el entrenador adopta un estilo de apoyo a la autonomía, su motivación para practicar deporte es más autodeterminada (mayores niveles de motivación intrínseca y regulación identificada especialmente). Actuar de este modo, significa favorecer que los bailarines funcionen de forma autónoma y con iniciativa personal, lo cual es importante en contextos como la danza donde la armonía y el ritmo se ven especialmente favorecidos con las formas más autodeterminadas de motivación (Balaguer et al, 2011).

Por otro lado, no se ha comprobado la relación positiva entre los índices de bienestar (autovalía global y percepción de competencia en danza) y las motivaciones autodeterminadas en danza (hipótesis 4). Los resultados van en la dirección inversa, indicando significaciones negativas en el caso de autovalía global y el continuo de autorregulación. No obstante, como se ha comentado anteriormente, se deben tomar precauciones al interpretar estos resultados, ya que el índice de fiabilidad del cuestionario de autoconcepto en danza no ha sido 
satisfactorio. Tras exhaustivas revisiones de los datos recogidos y análisis realizados, únicamente se podrían explicar los resultados por una mala comprensión del cuestionario. Las notas de campo tomadas por el investigador que realizó la administración, dan fe de que los bailarines tuvieron muchas dudas con el formato del cuestionario de Harter (autovalía global y autopercepción de competencia en danza), llegando a corregir sus propias respuestas. Por este motivo, sería interesante ahondar en este punto en una segunda fase de evaluación.

La última hipótesis sugerida en este estudio relaciona el clima de apoyo a la autonomía ofrecido por los profesores de danza y el bienestar, atendiendo a las variables autovalía global y percepción de competencia en danza (hipótesis 5). En base a la literatura (Balaguer et al, 2008; Adie et al, 2012; Balaguer, González, Fabra, Castillo, Mercé, y Duda, 2012; González et al, 2015) se esperaba encontrar correlaciones positivas. No obstante, la única significación hallada ha sido negativa (entre clima de apoyo a la autonomía y autovalía global). Consideramos que los motivos pueden ser los especificados en la hipótesis anterior.

$\mathrm{Si}$ atendemos a las puntuaciones brutas obtenidas en los factores estudiados, observamos que son elevadas. Destaca la alta percepción de apoyo a la autonomía en el centro, la satisfacción de las tres necesidades básicas y los niveles intrínsecos de motivación en danza.

En cuanto a las limitaciones del estudio, destaca la imposibilidad de establecer relaciones causales entre las variables de la SDT estudiadas. Se trata de un estudio transversal y correlacional en el que los resultados deben interpretarse con cautela. Estudios longitudinales permitirían inferir datos de forma más fiable y detectar cambios en las relaciones postuladas.

Como se ha apuntado anteriormente, uno de los instrumentos utilizados ha resultado confuso para algunos participantes. Se trata del Perfil de Autopercepción de Harter. A pesar de que durante el pase de cuestionarios se insistió en el formato correcto, muchas personas tuvieron que rehacerlo por una mala comprensión de los ítems. Esta dificultad es algo a tener en cuenta de cara a la interpretación de los datos obtenidos. Sería conveniente estudiar otras medidas de bienestar que ya han obtenido resultados muy interesantes tanto en deporte en general como en danza. Algunas variables interesantes en este punto son la vitalidad subjetiva (Quested y Duda, 2009c; Balaguer et al, 2011; Guzmán et al, 2012; González et al, 2015), la satisfacción con la vida (Torregrosa, Belando y Moreno-Murcia, 2014; González, Solá, Corte-Real; Fonseca, 2016) o la intención de seguir participando en la actividad (Duda y Balaguer, 2007; Balaguer et al, 2011). No se han analizado todas las relaciones posibles dentro del modelo de la SDT. En un futuro sería interesante completar la exploración averiguando las influencias entre otros factores como las relaciones entre los outputs de bienestar y la satisfacción de las necesidades psicológicas básicas.

En este trabajo se ha obtenido información relevante para conocer cómo afectan los aspectos motivacionales en el alumnado de la escuela de danza en cuestión. Este conocimiento puede ser de gran utilidad para la intervención aplicada sirviendo de primera fase de detección de necesidades. Para tener una idea más completa de la situación, podría compararse la visión de los bailarines, la de sus profesores de danza y la de su directora. También sería conveniente recoger las opiniones de los bailarines sobre cada profesor por separado 
y completar las medidas de autoinformes obtenidas con otras formas de evaluación como observación y entrevistas para comprender mejor algunos puntos clave.

Sería muy positivo que un psicólogo deportivo ofreciera charlas formativas a los profesores, asesorando empíricamente acerca de las consecuencias del clima con el que se trabaja en las sesiones y dando las claves necesarias para satisfacer las necesidades de autonomía, competencia y relación de su alumnado. La formación del profesorado en habilidades psicológicas es esencial para que acompañen a sus bailarines a alcanzar su máximo potencial artístico, cerciorarse de que la experiencia de la danza les parezca divertida y gratificante y crear un entorno que fomente su enriquecimiento personal y profesional.

Ayudar a los estudiantes desde una edad temprana a incrementar su conciencia de la independencia y promover la autorresponsabilidad en la danza es importante. La prevención en el desarrollo psicológico durante la etapa formativa es esencial, ya que una detección e intervención a tiempo puede evitar un mayor sufrimiento y un posible abandono de la actividad. La calidad de la experiencia deportiva proporcionada por el ambiente en el que se desarrolla la actividad, especialmente entrenadores o profesores, es la responsable de las implicaciones positivas para la consecución del bienestar de las personas (Duda et al, 2007).

Este estudio deja constancia de la importancia de trabajar bajo un clima positivo en las escuelas de danza: apoyando la autonomía del alumnado y favoreciendo la satisfacción de sus necesidades psicológicas y su motivación autorregulada.

\section{Referencias}

Adie, J. W., Duda, J. L. y Ntoumanis, N. (2008). Autonomy support basic need satisfaction and the optimal functioning of adult male and female sport participants: A test of basic needs theory. Motivation and Emotion, 32, 189-199.

Adie, J. W., Duda, J. L. y Ntoumanis, N. (2012). Perceived coach-autonomy support, basic need satisfaction and the well- and ill-being of elite youthsoccer players: A longitudinal investigation. Psychology of Sport and Exercise, 13, 51-59.

Amado, D., Leo. F. M., Sánchez, P.A., Sánchez, D. y García-Calvo, T. (2010). Importancia de los aspectos motivacionales sobre las estrategias de afrontamiento en practicantes de danza: Una perspectiva desde la teoría de Autodeterminación. Revista Iberoamericana de Psicología del Ejercicio y el Deporte, 5(2), 179-194.

Balaguer, I., González, L., Fabra, P., Castillo, I., Mercé, J., y Duda, J. L. (2012). Coaches' interpersonal style, basic psychological needs and the well-and ill-being of young soccer players: A longitudinal analysis. Journal of Sports Sciences, 30(15), 1619-1629.

Balaguer, I., Castillo, I. y Duda, J.L. (2008). Apoyo a la autonomía, Satisfacción de las Necesidades, motivación y Bienestar en deportistas de Competición: un análisis de la Teoría de la Autodeterminación. Revista de Psicología del Deporte. 17(1), 123-139.

Balaguer, I., Castillo, I. Duda, J.L y García-Merita, M. (2011). Asociaciones entre la percepción del clima motivacional creado por el entrenador, orientaciones disposicionales de meta, regulaciones motivacionales y vitalidad subjetiva en jóvenes jugadoras de tenis. Revista de Psicología del Deporte. 20(1), 133-148. 
Balaguer, I., Castillo, I., Duda, J.L., Quested, E., y Morales, V. (2011). Predictores socio-contextuales y motivacionales de la intención de continuar participando: un análisis desde la SDT en danza. Revista Internacional de Ciencias del Deporte, 25(7), 305-319.

Balaguer, I.; Castillo, I.; Duda, J.L., y Tomás, I. (2009). Análisis de las propiedades psicométricas de la versión española del Cuestionario de Clima en el Deporte. Revista de Psicología del Deporte, 18, 73-83.

Balaguer, I., y Pastor, Y. (2001). Un estudio acerca de la relación entre el autoconcepto y los estilos de vida en la adolescencia media. En CIOPA 2001-Congreso Internacional Online de Psicología Aplicada (pp. 11-18).

DeCharms, R. (1968). Personal causation: The Internal Affective Determinants of Behavior. Nueva York. Academic Press.

Deci, E.L. y Ryan, R.M. (1985). Intrinsic motivation and self-determination in human behavior. Nueva York: Plenum Press.

Deci E.L., Ryan R.M. (2000). The "what" and "why” of goal pursuits: human needs and the self-determination of behavior. Psychol Inq, 11(4), 227-68.

Duda, J. L. y Balaguer, I. (2007). Coach-created motivational climate. En S. Jowett y D. Lavallee (Eds.), Social psychology in sport (pp. 117-130). Champaign, IL: Human Kinetics.

González, L., Castillo, I., García-Merita, M. y Balaguer, I. (2015). Apoyo a la autonomía, satisfacción de las necesidades psicológicas y bienestar: Invarianza de un modelo estructural en futbolistas y bailarines. Revista de Psicología del Deporte, 24(1), 121-129.

González, F. L. A., Solá, I. B., Corte-Real, N., y Fonseca, A. M. (2016). Factorial invariance of the Satisfaction with Life Scale in adolescents from Spain and Portugal. Psicothema, 28(3), 353-358.

González, L., Tomás, I., Castillo, I., Duda, J. L., y Balaguer, I. (2016). A test of basic psychological needs theory in young soccer players: time-lagged design at the individual and team levels. Scandinavian Journal of Medicine \& Science in Sports.

Guzmán, J.F. y García-García, C. (2012). Bienestar Psicológico en Bailarines: un análisis social cognitivo. En Giménez, C. (Ed.) La investigación en Danza en España 2012. Valencia: Mahali.

Hair, J., Black, B., Babin, B., Anderson, R. y Tatham, R. (2006). Multivariate Data Analysis (6 ed.). Upper Saddle River, NJ: Prentice-Hall.

Harter, S. (1985). Manual for the Self-Perception Profile for Children. Denver: University of Denver.

Harter, S. (1999). The construction of the self: A developmental perspective. Nueva York: Guildford Press.

Harter, S. (2012). Self-Perception Profile for Children: Manual and Questionnaires (Grades 3/8). Revision of the Self-Perception Profile for Children, 1985. Denver: University of Denver.

Lonsdale, C.,Hodge, K., y Rose, E.A. (2008). The development of the Behavioral Regulation in Sport Questionnaire (BRSQ): Instrument development and initial validity evidence. Journal of Sport \& Exercise Psychology, 30, 323-355.

López-Walle, J., Balaguer, I., Castillo. I. y Tristán, J. (2012). Autonomy support, basic psychological needs and well-being in Mexican athletes. The Spanish Journal of Psychology, 15, 1283-1292.

McAuley, E., Duncan, T.yTammen, V.V. (1989). Psychometric properties of the intrinsic motivation inventory in a competitive sport setting: A confirmatory factor analysis. Research Quarterly for Exercise and Sport, 60(1), 48-58.

Nunnally, J.C. (1978). Psychometric theory. Nueva York: McGraw-Hill.

Pelletier, L.G., Fortier, M.S.; Vallerand, R.J. y Brière, N.M. (2001). Associations among perceived autonomy support, forms of Self-Regulation and Persistence: A Proespective Study. Motivation and Emotion, 25, 279-306.

Quested, E., Bosch, J., Burns, V.E., Cumming, J., Ntoumanis, N., y Duda, J.L. (2010). Hormonal and emotional responses to performance stress. En: The role of psychological need satisfaction. Self-Determination Theory Conference. Ghent, Belgium.

Quested, E., y Duda, J. L. (2009a). Maintaining the balance: Striving for quality and quantity in the growing field of dance psychology. IADMS newsletter. 
Quested, E., y Duda, J. L. (2009b). Perceptions of the motivational climate, need satisfaction, and indices of well- and illbeing among hip hop dancers. Journal of Dance Medicine and Science, 13(1), 10-19.

Quested, E., y Duda, J. L. (2009c). Setting the stage: social-environmental and motivational predictors of optimal training engagement. Performance Research,14(2), 36-45.

Quested,E., y Duda,J.L. (2010).Exploring the social-environmental determinants of welland ill-being in dancers: A test of Basic Needs Theory. Journal of Sport and Exercise Psychology, 32(1), 39-60.

Quested, E., y Duda, J.L. (2011). Antecedents of burnout among elite dancers: a longitudinal test of basic needs theory. Psychology of Sport and Exercise, 12(2), 159-167.

Quested, E., Duda, J.L., Ntoumanis, N., y Maxwell, J.P. (2013). Daily fluctuations in the affective states of dancers: A crosssituational test of basic needs theory. Psychology of Sport and Exercise, 14, 586-595.

Reeve, J. (2009). Why teachers adopt a controlling motivating style toward students and hoy they can become more autonomy supportive. Educational Psychologist, 44, 159-175.

Ryan, R.M., y Deci, E.L. (2002). Overview of Self-Determination Theory: An organismic dialectical perspective. En Deci, E.L. y Ryan, R.M. (Eds.), Handbook of Self Determination Research (pp. 3-33). Rochester: The University of Rochester Press.

Reinboth, M. y Duda, J.L. (2006). Perceived motivational climate, need satisfaction and indices of well-being in team sports: A longitudinal perspective. Psychology of Sport and Exercise, 7, 269-286.

Richer, S. y Vallerand, R. J. (1998). Construction and validation of the Perceived Relatedness Scale. Revue Européene de Psychologie Appliquée, 48, 129-137.

Ryan, R.M.,y Deci, E.L.(2000a). Self-determination theory and the facilitation of intrinsicmotivation, social development, and well-being. American Psychologist, 55, 68-78.

Ryan, R.M., y Deci, E.L. (2000b). The darker and brighter sides of human existence: basic psychological needs as a unifying concept. Psychological Inquiry, 11(4), 319-338.

Ryan, R.M., y Deci, E.L. (2002). Overview of Self-Determination Theory: An organismic dialectical perspective. En Deci, E.L. y Ryan, R.M. (Eds.), Handbook of Self Determination Research (pp. 3-33). Rochester:The University of Rochester Press.

Ryan, R.M., y Deci, E.L. (2007). Active human nature: Self-determination theory and the promotion, and maintenance of sport, exercise and health. Hagger MS.

Sport Climate Questionnaire (n.d.). Extraído en Junio de 2003 del sitio Web de la Self-Determination Theory (SDT): http://www.psych.rochester.edu/SDT/measures-/auton_sport.html

Taylor, J. y Taylor, C. (2008). Psicología de la Danza. Madrid: Gaia Ediciones.

Torregrosa, D., Belando, N. y Moreno-Murcia, J. A. (2014). Predicción de la satisfacción con la vida en practicantes de ejercicio físico saludable. Cuadernos de Psicología del Deporte, 14(1), 117-122.

White R.W. (1959). Motivation reconsidered: the concept of competence. Psychol Rev. 66, 297-333.

Williams, G. C., Grow, V. M., Freedman, Z., Ryan, R. M. y Deci, E. L. (1996). Motivational predictors of weight loss and weight-loss maintenance. Journal of Personality, 70, 115-126. 